\title{
Hypertrophic cardiomyopathy without hypertrophy: two families with myocardial disarray in the absence of increased myocardial
} mass

\author{
W J McKenna, J T Stewart, P Nihoyannopoulos, F McGinty, M J Davies
}

\begin{abstract}
Two families are described in which individuals showed widespread myocardial disarray at histological examination, in the absence of macroscopic cardiac hypertrophy. In one family the clinical presentation was that of sudden unexpected cardiac death in four family members; members of the other family presented with electrocardiographic repolarisation changes and abnormalities of left ventricular diastolic function.

The finding of myocardial disarray, the characteristic histological abnormality of hypertrophic cardiomyopathy, in the absence of increased cardiac mass suggests a wider range of abnormality in hypertrophic cardiomyopathy than is currently recognised.
\end{abstract}

The most characteristic abnormalities of hypertrophic cardiomyopathy are an increase in ventricular muscle mass with myocyte hypertrophy and disarray. ${ }^{1-3}$ The criteria for the diagnosis of hypertrophic cardiomyopathy and the nomenclature for its description have expanded since Teare's original description of these abnormal features. ${ }^{1-4}$

In the 1960s the diagnosis relied on clinical and haemodynamic features of a left ventricular outflow tract pressure drop (hypertrophic obstructive cardiomyopathy, idiopathic hypertrophic subaortic stenosis, muscular subaortic stenosis) ${ }^{235}$; it is now known that only about $35^{\circ}{ }_{0}$ of patients have left ventricular outflow tract pressure differences at rest. ${ }^{6}$ The development of $M$ mode echocardiography in the 1970 s permitted the visualisation of the interventricular septum and the posterior left ventricular wall at mitral valve level. ${ }^{78} \mathrm{M}$ mode echocardiography confirmed earlier observations and led to the assertion that asymmetrical septal hypertrophy was the pathognomonic feature of the condition. ${ }^{9}$ Asymmetrical septal hypertrophy is seen in $60-70 \%$ of patients with hypertrophic cardiomyopathy but it is also found in other patient groups. ${ }^{10}$ Currently the clinical diagnosis of hypertrophic cardiomypathy is based on the demonstration of unexplained left ventricular hypertrophy when the entire heart is visualised by cross sectional echocardiography. ${ }^{11} 12$

The extent of myocyte disarray is now regar- ded as a specific histological marker of hypertrophic cardiomyopathy. In normal hearts the mean proportion of the septum with disarray is $1.5^{\circ}$; in hypertrophic cardiomyopathy disarray is found in $30 \%$ of the total tissue area. ${ }^{1314}$ The histological appearances of disarray are classified as type I-in which the myocytes are predominantly cut longitudinally-and type II-in which the myocytes are cut transversely. The degree of disarray on histological slides is expressed as a proportion of the total area cut longitudinally or transversely, and the largest percentage is used to indicate the amount of disarray. ${ }^{1314}$

We report two families with myocyte disarray but no increase in cardiac mass. These families raise questions about the appropriateness of current nomenclature and highlight potential limitations of current diagnostic criteria.

\section{Clinical and pathological details}

FAMILY A

This family came to attention because of a strong history of premature sudden death. The proband (case 1) died at the age of 38 years with no previous history of cardiac disease. Her daughter (case 2), sister (case 3), and nephew (case 4) (aged 21, 42, and 17 years respectively) also died suddenly and unexpectedly without any previous history to suggest cardiac abnormality (fig 1).

Necropsy was performed on all 4. Table 1 gives detailed pathological information. The macroscopic appearances of the hearts were normal. Heart weights were not increased; cavity size and ventricular wall thickness were normal. Histological assessment, however, showed widespread interstitial fibrosis with

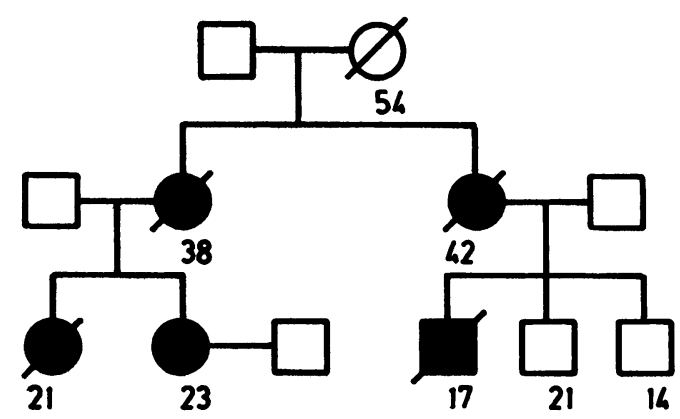

Figure 1 Pedigree of family A. Solid symbols represent affected individuals. Age at death or diagnosis is shown.

London SW 17 ORE.

Accepted for publication 10 November 1989 
Table 1 Results of pathological examination in five individuals showing considerable myocardial disarray

\begin{tabular}{|c|c|c|c|c|c|c|}
\hline Patient & Age/sex & Cause of death & Heart weight ( $g$ ) & $L V$ mass $(g)$ & $R V$ mass $(g)$ & Disarray $\left({ }^{\circ}{ }_{0}\right)$ \\
\hline \multicolumn{7}{|l|}{ Family A: } \\
\hline & $38 / \mathrm{F}$ & SCD & 374 & - & - & $61 \cdot 3$ \\
\hline 2 & $21 / F$ & SCD & 292 & 136 & 42 & $32 \cdot 8$ \\
\hline 3 & $42 / F$ & SCD & - & - & - & $36 \cdot 0$ \\
\hline 4 & $17 / M$ & SCD & 341 & 163 & 47 & $47 \cdot 0$ \\
\hline 5 & $44 / F$ & MI & 325 & 164 & 44 & $25 \cdot 6$ \\
\hline
\end{tabular}

LV, left ventricular; MI, myocardial infarction; RV, right ventricular; SCD, sudden cardiac death.

type I disarray in three (myocytes predominantly cut longitudinally). In patient 3 there was diffuse fibrosis with $36 \%$ disarray on transverse section (type II) in the only tissue sample available for review from the original necropsy (fig 2).

The three surviving first degree relatives were symptom free. Two were willing to be assessed. Clinical examination was normal in both, and in one the electrocardiogram and cross sectional echocardiogram were also normal. In the surviving daughter (case 6), of the proband, however, the resting electrocardiogram was abnormal, and fulfilled voltage criteria for left ventricular hypertrophy, with associated widespread abnormalities of repolarisation. This woman provides an opportunity to study her family's cardiac abnormality in life. Her cross sectional echocardiogram showed normal cavity size, wall thickness, and functional indices (left ventricular end systolic dimension $26 \mathrm{~mm}$, left ventricular end diastolic dimension $40 \mathrm{~mm}$, interventricular septal thickness $9 \mathrm{~mm}$, posterior left ventricular wall thickness $9 \mathrm{~mm}$ ) (fig 3). She exercised for 12 minutes of a standard Bruce protocol; her heart rate increased from 60 to 167 beats/min, with maximal oxygen consumption $\left(\dot{\mathrm{V}}_{2} \mathrm{max}\right)$ of $40 \mathrm{ml} / \mathrm{kg} / \mathrm{min}(100 \%$ of her predicted maximum). The $\mathrm{T}$ wave inversion in the anterior chest leads of the resting electrocardiogram became normal during exercise. The blood pressure response to exercise,

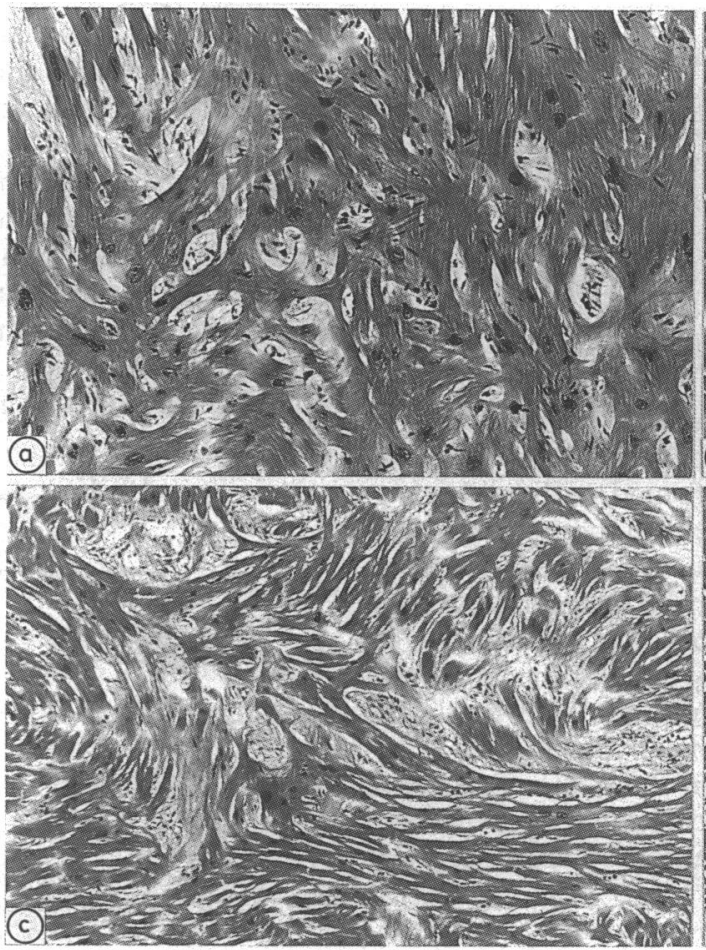

however, was abnormal; the resting systolic pressure was $100 \mathrm{~mm} \mathrm{Hg}$, it rose early during exercise to $125 \mathrm{~mm} \mathrm{Hg}$, and fell to $90 \mathrm{~mm} \mathrm{Hg}$ at peak exertion. The control of the peripheral vasculature, investigated by forearm plethysmography during supine bicycle exercise, was abnormal with an increase in forearm blood flow indicating inappropriate and generalised vasodilatation. A high gain signal averaged electrocardiogram did not show late potentials and there were no arrhythmias during repeated 48 hour electrocardiographic monitoring. The findings on left and right cardiac catheterisation (including filling pressures) were normal and there was no resting or inducible outflow tract gradient in the left or right ventricle. At electrophysiological study the basic conduction intervals were normal and there was no evidence of an accessory atrioventricular pathway or of accelerated atrioventricular nodal conduction. Atrial vulnerability was assessed by high rate burst pacing and extrastimuli, and ventricular vulnerability by programmed stimulation to stage 8 of the Wellens protocol (drive cycle $420 \mathrm{~ms}$ and 2 extrastimuli), but tachycardia was not initiated.

She was treated with low dose amiodarone (200 mg daily, plasma concentration $0.8 \mathrm{mg} / \mathrm{l}$ ), and has remained well during four years of follow up.

FAMILY B

In this family the proband (case 5) died of
Figure 2 (a)

Disorganisation of cardiac muscle cells that are aligned obliquely (type I) to each other forming tangled masses (case 1). (b) Disorganisation showing whorled arrangement (type I) of cardiac muscle cells around a central focus of connective tissue (case 1). (c) Disorganisation in which bundles of cardiac muscle cells are arranged at oblique angles (type I) (case 2). (d)

Disorganisation with narrow bands of longitudinally (type II) arranged myocytes alternating with transversely cut myocytes (case 3).

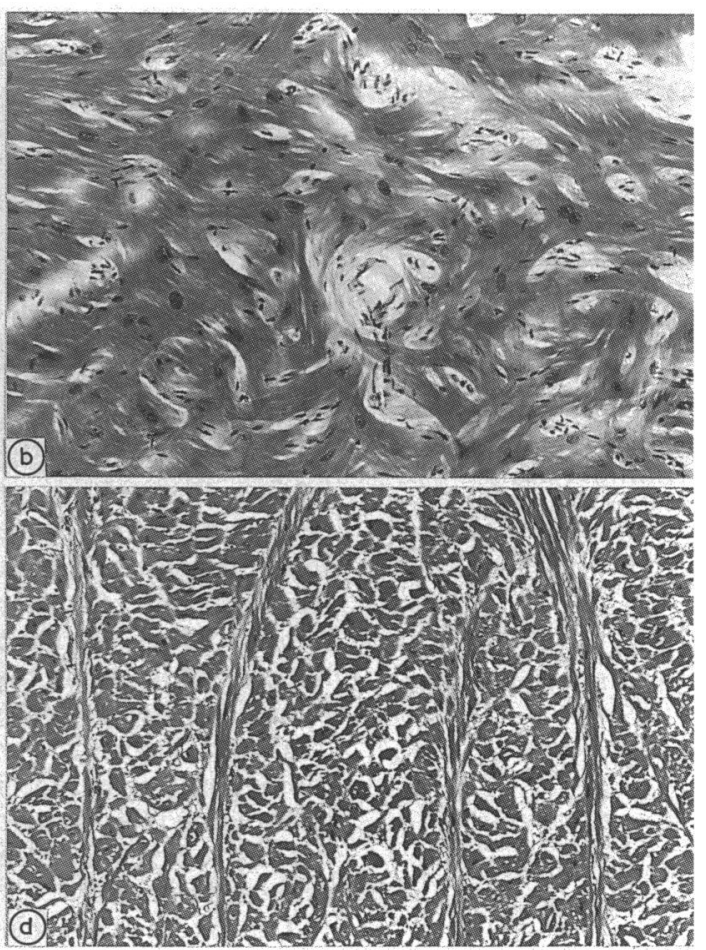


Figure 3 Cross sectional echocardiogram and 12 lead surface electrocardiogram of case 6 $($ family $A)$.

Figure 4 Pedigree of family $B$. Solid symbols represent affected individuals. Age at death or diagnosis is shown.
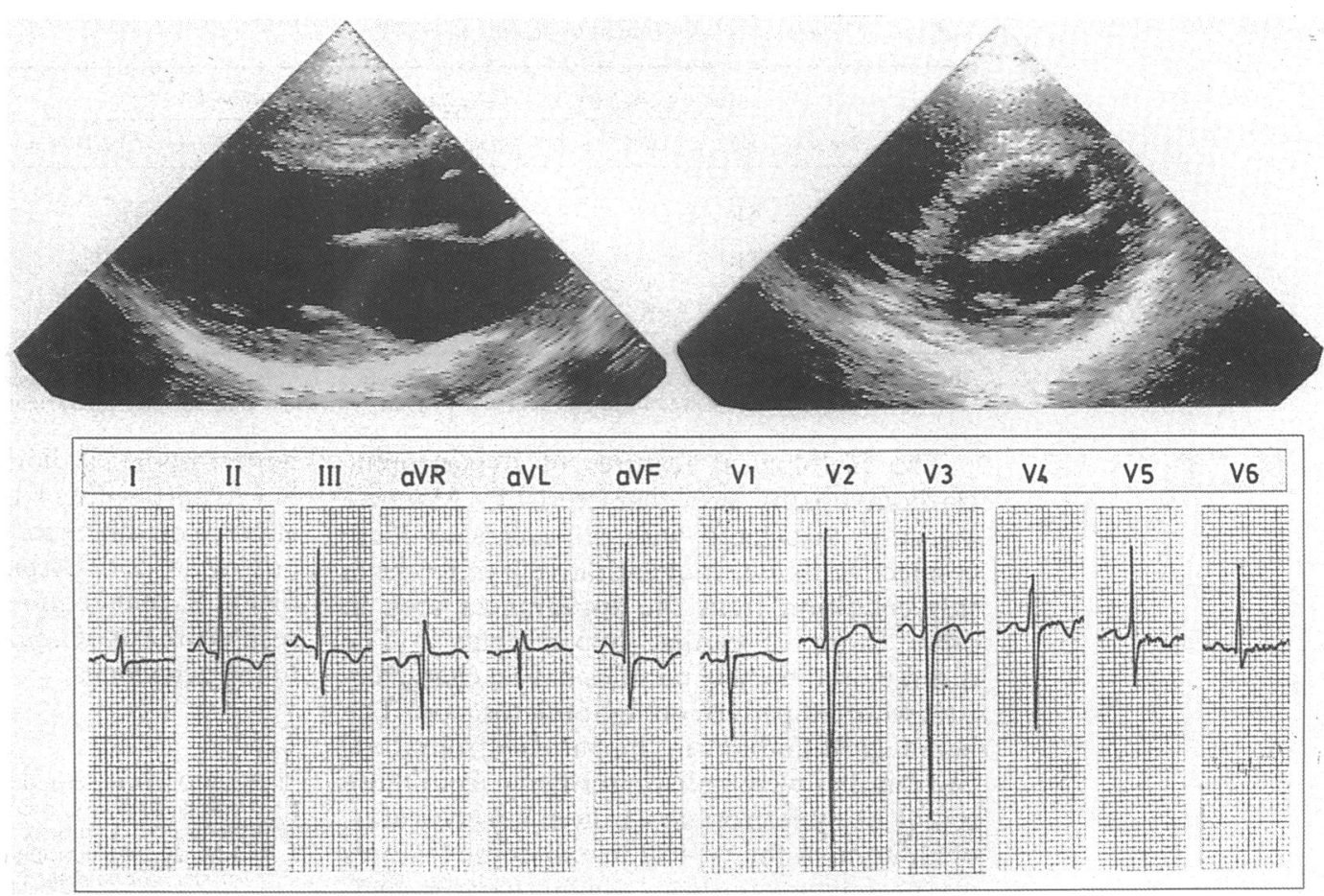

myocardial infarction after coronary arteriography in 1979. She had a 5 year history of paroxysmal atrial fibrillation and an abnormal resting electrocardiogram showing atrial overload, voltage criteria of left ventricular hypertrophy, and widespread repolarisation abnormalities. Six months before her death she started to complain of chest tightness and dyspnoea on exertion, which were relieved by rest and sublingual glyceryl trinitrate. $M$ mode echocardiography at that time showed a small left ventricle (end diastolic dimension $41 \mathrm{~mm}$, end systolic $29 \mathrm{~mm}$ ) with wall thickness at the upper limit of normal (interventricular septum $12 \mathrm{~mm}$, posterior left ventricular wall $13 \mathrm{~mm}$ ), and a restrictive filling pattern. The left atrial dimension was also at the upper limit of normal $(40 \mathrm{~mm})$. Left ventricular cineangiography showed normal systolic function and coronary arteriography showed some irregularity of the left main stem coronary artery. During the next 6 months she had progressive angina despite treatment with propranolol; nitrates, and verapamil. At restudy when the right coronary arteriogram was performed (before cannulation of the left coronary artery) the entire left coronary system was seen to fill retrogradely. After this she suffered an extensive anterior myocardial infarct and died 24 hours later in cardiogenic shock.

The clinical diagnosis was severe stenosis of the left main stem coronary artery. At necropsy

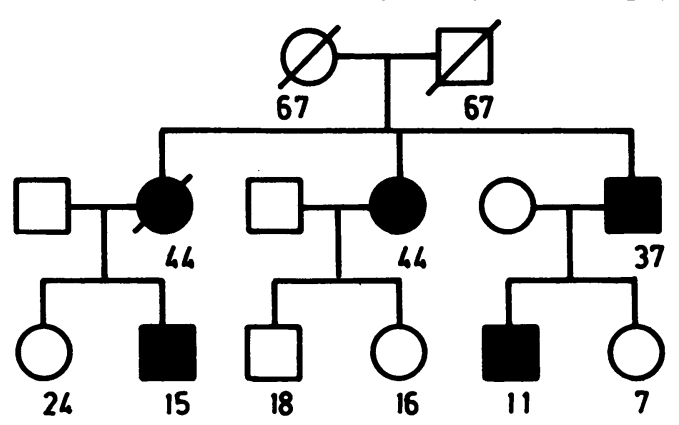

a small plaque of coronary atheroma was found in the proximal left main stem coronary artery; there was no significant atheroma elsewhere in the coronary tree. The infarct that caused her death was assumed to have been caused by spasm at the site of the plaque in her left main coronary artery. Otherwise the heart was macroscopically normal, apart from unusual rigidity of the atria, and weighed $325 \mathrm{~g}$ (normal for her body weight). Histological examination showed extensive interstitial fibrosis of the atria with obliteration of the sinoatrial node. The ventricular myocardium showed less pronounced fibrotic changes but severe myocyte disarray $\left(25 \cdot 6^{\circ}{ }_{0}\right.$ type I).

Eight first degree relatives of this patient, all of whom were symptom free, were assessed (fig 4). Four had abnormal electrocardiograms and cross sectional echocardiograms. All four showed abnormalities of ventricular repolarisation on the resting 12 lead electrocardiogram; atrial fibrillation was also seen in one, and left or right atrial overload or both was seen in the other three. The echocardiograms in these four patients showed normal heart size, ventricular cavity dimensions, and ventricular wall thickness. The ventricular filling patterns, however, showed a phase of early rapid filling that stopped abruptly in mid-diastole. In addition, the echocardiogram confirmed the presence of atrial enlargement in three of these four (table 2).

\section{Discussion}

Familial hypertrophic cardiomyopathy shows a mendelian autosomal dominant pattern of inheritance. ${ }^{15}$ The pattern of inheritance in the two families described is consistent with autosomal dominance-if one accepts that the survivors with electrocardiographic or echocardiographic abnormalities or both have the same condition as those examined at necropsy. 
Table 2 Clinical details of surviving family members

\begin{tabular}{|c|c|c|c|c|c|c|c|c|c|c|c|c|}
\hline \multirow[b]{2}{*}{ Patient } & \multirow[b]{2}{*}{ Age/sex } & \multicolumn{6}{|c|}{ Electrocardiogram } & \multicolumn{5}{|c|}{ Echocardiogram } \\
\hline & & Rhythm & $R A+$ & $L A+$ & $T a b n$ & $S T a b n$ & $L V H$ & $L V W T(\mathrm{~mm})$ & $L V E D(\mathrm{~mm})$ & LVES ( $\mathrm{mm})$ & $L A(\mathrm{~mm})$ & $E F\left({ }^{\circ}{ }_{0}\right)$ \\
\hline $\begin{array}{l}\text { Family A: } \\
6 \\
\text { Family B: }\end{array}$ & $25 / F$ & SR & - & - & + & - & + & 10 & 35 & 20 & 24 & 63 \\
\hline $\begin{array}{l}7 \\
8 \\
9\end{array}$ & $\begin{array}{l}15 / M \\
11 / M \\
44 / F\end{array}$ & $\begin{array}{l}\text { SR } \\
\text { SR } \\
\text { AF }\end{array}$ & $\begin{array}{l}+ \\
+ \\
+\end{array}$ & $\overline{-}$ & $\overline{+}$ & $\frac{t}{+}$ & \pm & $\begin{array}{r}9 \\
8 \\
10\end{array}$ & $\begin{array}{l}43 \\
40 \\
43\end{array}$ & $\begin{array}{l}26 \\
27 \\
32\end{array}$ & $\begin{array}{l}45 \\
42 \\
46\end{array}$ & $\begin{array}{l}70 \\
68 \\
47\end{array}$ \\
\hline 10 & $37 / M$ & SR & - & + & - & - & + & 10 & 49 & 33 & 39 & 63 \\
\hline
\end{tabular}

Abn, abnormality; AF, atrial fibrillation; EF, ejection fraction; LA, left atrial; LVED, left ventricular end diastolic dimension;

LVES, left ventricular end systolic dimension; LVWT, left ventricular wall thickness; RA, right atrial; SR, sinus rhythm.

The histological features of hypertrophic cardiomyopathy are characteristic. Myocyte disarray, associated with an excess of loose connective tissue, is quantitatively different in this condition from the appearances seen in other forms of cardiac hypertrophy. ${ }^{1-3}$ The disorganised muscle bundles have a characteristic whorled pattern on light microscopy. The histological findings in the patients from these two families were indistinguishable from those found in hypertrophic cardiomyopathy, although none of the typical macroscopic features was present and the heart weights were normal.

One of the possible consequences of myocyte disarray is electrical instability of the myocardium, which allows the development of ventricular fibrillation but does not provide the basis for sustained ventricular tachycardia (this arrhythmia is very rare in hypertrophic cardiomyopathy). ${ }^{16}$ The exact mechanism of sudden cardiac death in hypertrophic cardiomyopathy is still unresolved. Based on our studies in this condition, we believe that the primary event is most often haemodynamic collapse resulting from inappropriate circulatory reflexes. ${ }^{1718}$ The resulting hypotension and ischaemia may provoke ventricular fibrillation in individuals with severe disarray. Investigation of the surviving member of family A did not show any evidence of electrical instability but did suggest abnormal control of the circulation during exercise.

Myocyte disarray may also contribute to abnormalities of left ventricular diastolic function seen in hypertrophic cardiomyopathy. In Family $B$ restrictive physiology was seen in surviving family members, although only the proband showed histological proof of myocyte disarray. The explanted hearts of five young patients (age $<15$ years) undergoing transplantation for restrictive cardiomyopathy, which were examined recently by us, showed similar histological features (A Pomerance, personal communication).

Did the patients described have hypertrophic cardiomyopathy without hypertrophy? If they do represent a previously unrecognised form of the condition then, by implication, the range of disease recognised by standard diagnostic echocardiographic criteria is limited. More extensive characterisation of similar patients may confirm our impression that hypertrophic cardiomyopathy may exist without hypertrophy or that within a family some affected members may have hypertrophy while others do not. Ultimately, however, accurate diagnosis may require identification of the gene responsible or of a genetic marker that is linked to the gene locus.

1 Teare D. Asymmetrical hypertrophy of the heart in young adults. Br Heart J 1958;20:1-8.

2 Braunwald E, Lambrew C, Rockoff S, Ross J, Morrow AG. Idiopathic hypertrophic subaortic stenosis. I. Description of the disease based upon an analysis of 64 patients. Circulation 1964;29(suppl 4):III-119.

3 Cohen J, Effat H, Goodwin JF, Oakley CM, Steiner RE. Hypertrophic obstructive cardiomyopathy. Br Heart $J$ 1964;26:16-32.

4 Maron BJ, Epstein SE. Hypertrophic cardiomyopathy: a discussion of the nomenclature. Am J Cardiol 1979; 43:1242-4.

5 Wigle ED, Helmbecker RO, Gunton RW. Idiopathic ventricular septal hypertrophy causing muscular subaortic stenosis. Circulation 1962;26:325-40.

6 Maron BJ, Bonow RO, Cannon RO III, Leon MB, Epstein SE. Hypertrophic cardiomyopathy. Interrelations of clinical manifestations, pathophysiology and therapy. (Parts 1 and 2). N Engl J Med 1987;316:780-9 and 844-52.

7 Abbasi AS, MacAlpin RN, Eber LM, Pearce ML. Left ventricular hypertrophy diagnosed by echocardiography. ventricular hypertrophy diagnosed

8 Tajik AJ, Giuliani ER. Echocardiographic observations in idiopathic hypertrophic subaortic stenosis. Mayo Clin Proc 1974;49:89-97.

9 Henry WL, Clark CE, Epstein SE. Asymmetric septal hypertrophy: echocardiographic identification of the pathognomonic anatomic abnormalities of IHSS. Circulation 1973;47:225-33.

10 Maron BJ, Epstein SE. Hypertrophic cardiomyopathy: recent observations regarding the specificity of three hallmarks of the disease: asymmetric septal hypertrophy,
septal disorganisation and systolic anterior motion of the anterior mitral leaflet. Am J Cardiol 1980;45:141-54

11 Maron BJ, Gottdiener JS, Epstein SE. Patterns and significance of distribution of left ventricular hypertrophy in hypertrophic cardiomy hypertrophic cardiomyopathy: a wide angle, two dimenCardiol 1981;48:418-28.

12 Shapiro LM, McKenna WJ. Distribution of left ventricular hypertrophy in hypertrophic cardiomyopathy: a twodimensional echocardiographic study. J Am Coll Cardiol 1983;2:437-44.

13 Maron BJ, Roberts WC. Quantitative analysis of cardiac muscle cell disorganisation in the ventricular septum of patients with hypertrophic cardiomyopathy. Circulation 1979;59:689-706.

14 Davies MJ. The current status of myocardial disarray in hypertrophic cardiomyopathy. Br Heart J 1984;51:361-3.

15 Clark CE, Henry WL, Epstein SE. Familial prevalence and genetic transmission of idiopathic hypertrophic subaortic stenosis. N Engl J Med 1973;289:709-14.

16 Alfonso F, Frenneaux MP, McKenna WJ. Clinical sustained uniform ventricular tachycardia in hypertrophic caruniform ventricular tachycardia in hypertrophic car-
diomyopathy: association with left ventricular apical diomyopathy: association with left
aneurysm. Br Heart J 1989;61:178-81.

17 Frenneaux MP, Counihan PJ, Webb D, McKenna WJ. Evidence for an abnormal vasodilator response in hyperEvidence for an abnormal vasodilator response in hyper-
trophic cardiomyopathy [Abstract]. J Am Coll Cardiol trophic cardiom

18 Counihan PJ, Frenneaux MP, O'Donoghue A, Webb D, McKenna WJ. Abnormal peripheral vascular response in hypertrophic cardiomyopathy in relation to clinical and prognostic features [Abstract]. Br Heart J 1989;61:456. 